\title{
49. Plaies et cicatrisation
}

(C) Springer-Verlag France 2010

\section{4}

\section{Hématomes des membres inférieurs chez la personne âgée ; diagnostic et traitement local \\ R. Van Den Bulck ${ }^{1}$ \\ ${ }^{1}$ Clinique Edith-Cavell, Bruxelles, Belgique}

Notre activité hospitalière nous a amenés à être confrontés aux conséquences cutanées des hématomes. Les hématomes chez la personne âgée sont souvent banalisés, voire négligés or les conséquences peuvent être sévères.

La prise d'un traitement antithrombotique, le caractère chuteur et le mauvais état nutritionnel de la personne âgée semblent être les trois facteurs favorisants les plus importants.
La prise en charge rapide de ces hématomes de gravité variable, permet d'éviter diverses complications cutanées secondaires dont la gravité dépend de l'importance de l'hématome.

Les hématomes disséquants exigent une évaluation de l'étendue de l'hématome, de l'œdème et de l'état des tissus péri lésionnels afin d'établir une prise en charge optimale. Cette prise en charge sera faite par une équipe multidisciplinaire étant donné que dans de nombreux cas un débridement chirurgical est nécessaire avant de traiter la plaie. Les différentes options de traitement local à l'aide de pansements ou par la pression négative topique auront pour objectif principal la préparation du lit de la plaie avant la greffe de peau

Dans cette présentation, nous insisterons sur l'importance de la chronologie de la prise en charge, la sélection du traitement local et l'importance d'un diagnostic précoce afin d'éviter des complications relatives aux hématomes. 